\title{
Mortalidad por fibrosis quística en Chile (1997-2003)
}

\author{
Jaime Cerda $L^{1}$, Gonzalo Valdivia $C^{1}$, Ernesto Guiraldes $C^{2}$, \\ Ignacio Sánchez $D^{3}$. \\ Cystic fibrosis mortality in Chile \\ between 1997 and 2003
}

Background: Cystic fibrosis (CF) is the most common lethal autosomic recessive disease among Caucasians. In Chile, its incidence is estimated in 1/4,000 newborns and it is possibly underestimated. Aim: To analyze CF mortality in Chile during the period 1997-2003. Material and methods: Demographic and CF mortality data reported by the National Institute of Statistics during the period 1997-2003 were recorded, according to sex and age. Overall mortality rate for each year was estimated, as well as the average mortality rate during the same period in patients younger than 1 year, 1-4 years, 5-9 years, 10-14 years and older than 15 years. Results: One hundred and three deaths (56 females) due to CF occurred during 1997-2003. Sixty-eight deaths corresponded to patients younger than 15 years (66.0\%). Overall mortality rate ranged from 0.82 to 1.33 per $10^{6}$ inhabitants in 1997 and 1999, respectively. Average mortality rate ranged from 0.46 to 9.81 per $10^{6}$ inhabitants among patients older than 15 years and younger than 1 year, respectively. Conclusions: Most CF deaths occurred in the pediatric age group (Rev Méd Chile 2008; 136: 157-62).

(Key words: Cause of death; Cystic fibrosis; Infant mortality)

Recibido el 20 de junio, 2007. Aceptado el 24 de septiembre, 2007.

${ }_{1}^{1}$ Departamento de Salud Pública. Facultad de Medicina, Pontificia Universidad Católica de Chile. Santiago, Chile. ${ }^{2}$ Departamento de Pediatría, Sección Gastroenterología y Nutrición Pediátrica, Facultad de Medicina, Pontificia Universidad Católica de Chile. Santiago, Chile. ${ }^{3}$ Departamento de Pediatría, Sección Respiratorio Pediátrico, Facultad de Medicina, Pontificia Universidad Católica de Chile. Santiago, Chile.

L a fibrosis quística (FQ) es una enfermedad de herencia autosómica recesiva, evolución crónica progresiva y compromiso multisistémico, con importantes variaciones fenotípicas y diferentes prevalencias en los diversos grupos étnicos. Diversas mutaciones del gen que codifica la proteína CFTR (cystic fibrosis transmembrane regulator)

Correspondencia a: Dr. Jaime Cerda L. Departamento de Salud Pública. Facultad de Medicina. Pontificia Universidad Católica de Chile. Marcoleta 434, Santiago Centro, Santiago-Chile. Tel: (56-2) 3543014. Fax: (56-2) 6331840. E mail. jcerda@med.puc.cl alteran su normal funcionamiento, produciendo, entre otras, infecciones respiratorias recurrentes e insuficiencia pancreática exocrina que caracterizan a la gran mayoría de los individuos que tienen esta enfermedad ${ }^{1}$.

Epidemiológicamente, la FQ es la enfermedad autosómica recesiva letal más frecuente en la raza caucásica. Su incidencia en Norteamérica y Europa Occidental es alrededor de $1 / 2.000$ a $1 / 2.500$ nacidos vivos, habiendo sido $50 \%$ de los pacientes diagnosticados ya a la edad de 6 meses y $90 \%$ a la edad de 8 años en los Estados Unidos de Norteamérica, en 20032. En Chile, su incidencia se estima en 1/4.000 
nacidos vivos ${ }^{3}$. Sin embargo, este dato es sólo una aproximación extrapolada de datos sobre la composición étnica de la población chilena, suponiéndose la existencia de un importante subdiagnóstico. En términos de mortalidad, en 1997 la Clasificación Internacional de Enfermedades (CIE-9) incluyó un código específico para $\mathrm{FQ}$, permitiendo el registro de todas sus defunciones según sexo y grupos de edad. La creación del Programa Nacional de Fibrosis Quística ${ }^{4}$ a cargo del Ministerio de Salud de Chile ha permitido registrar el número de pacientes con FQ atendidos exclusivamente en el sistema público de salud, contabilizándose para fines de 2003 un total de 270 pacientes reclutados. Sin embargo, hasta la fecha no se dispone de estudios que describan la mortalidad por FQ en pacientes chilenos. Con el objetivo de responder esta intermogante, se cuantificó y caracterizó la mortalidad por FQ en nuestro país durante el período 1997-2003.

\section{MATERIAL Y MÉTODO}

Los datos de población y defunciones por FQ durante el período 1997-2003 se obtuvieron de los anuarios demográficos publicados por el Instituto Nacional de Estadísticas. Se registraron las defunciones clasificadas bajo los códigos 277.0 (CIE-9) y E84 (CIE-10), correspondientes a "fibrosis quística", describiéndose su distribución porcentual según sexo y grupos de edad. Se calculó la tasa cruda de mortalidad por FQ para cada año del período 19972003 y la tasa de mortalidad promedio para el periodo 1997-2003 correspondiente a los siguientes grupos de edad: menores de 1 año, 1-4 años, 5-9 años, 10-14 años y mayores de 15 años durante igual período. Los datos fueron tabulados y analizados estadísticamente utilizando el programa Excel ${ }^{\circledR}$.

\section{RESULTADOS}

Durante el período 1997-2003 se registraron 103 defunciones por FQ. De ellas, $47(45,6 \%)$ correspondieron a varones y $56(54,4 \%)$ a mujeres. Sesenta y ocho defunciones ocurrieron en menores de 15 años (66,0\%), siendo éste el grupo de edad con el mayor número de defunciones en términos absolutos. Dentro de este grupo, correspondiente a la edad pediátrica, 24 defunciones ocurrieron entre los 5 y 9 años (35,3\%), dando cuenta del mayor porcentaje de casos, seguido por los menores de 1 año (26,5\%). La Tabla 1 resume la distribución de las defunciones según edad en términos absolutos y porcentuales.

La tasa bruta de mortalidad presentó su valor mínimo en 1997 y máximo en 1999 (0,82 y 1,33 por $1.000 .000 \mathrm{~h}$, respectivamente). La Figura 1 ilustra la evolución temporal del número de defunciones en términos absolutos, cuyo rango fluctúa entre 12 y 20 defunciones por año. Por su parte, la tasa de mortalidad promedio para cada grupo de edad durante el período 1997-2003, registró su valor mínimo en los mayores de 15 años y máximo en el grupo de menores de 1 año ( 0,46 y 9,81 por $1.000 .000 \mathrm{~h}$, respectivamente). La Figura 2 ilustra las tasas de mortalidad promedio para los distintos grupos de edad.

Tabla 1. D efunciones y tasa de mortalidad promedio por FQ, según grupos de edad (C hile, 1997-2003)

\begin{tabular}{|lcc|}
\hline Edad (años) & $\mathrm{N}(\%)$ & TMP (x 106) \\
\hline Edad pediátrica (0-14) & $68(66,0)$ & 2,24 \\
$\quad$ Menores de 1 & $18(26,5)$ & 9,81 \\
$1-4$ & $12(17,6)$ & 1,45 \\
$5-9$ & $24(35,3)$ & 2,35 \\
$10-14$ & $14(20,6)$ & 1,40 \\
Edad adulta $(15$ o +) & $35(34,0)$ & 0,46 \\
Total & $103(100,0)$ & 0,97 \\
\hline
\end{tabular}

TMP=tasa de mortalidad promedio (1997-2003). 


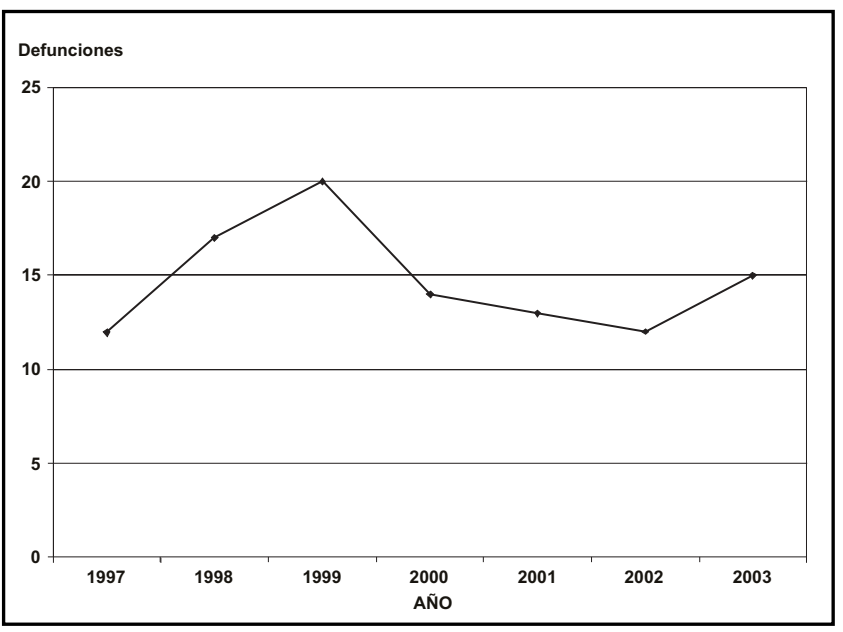

Figura 1. Número de defunciones por FQ (Chile, 1997-2003).

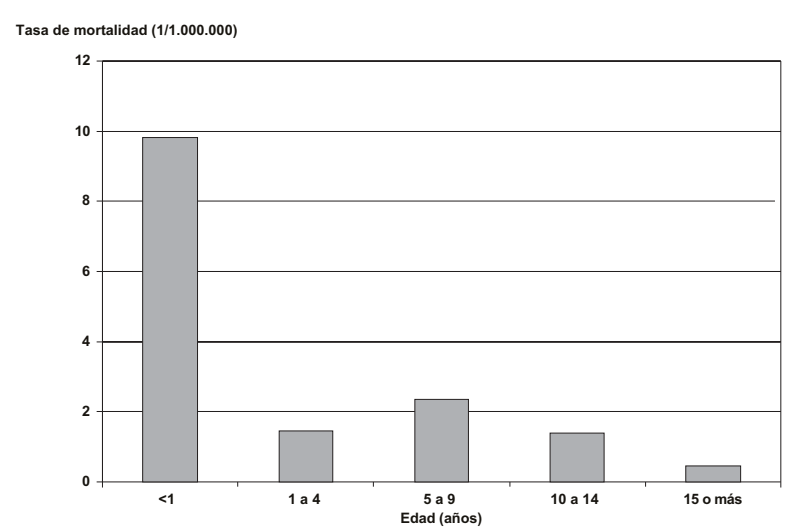

Figura 2. Tasa de mortalidad por FQ, según grupos de edad (Chile, 1997-2003).

\section{DisCUSIÓN}

El presente trabajo corresponde al primer reporte de mortalidad por FQ en Chile, analizada según sexo y grupo de edad. Internacionalmente, a fines de la década 1940-49, el Reino Unido y Dinamarca fueron pioneros en el registro de estadísticas vitales de pacientes con $F Q$, reportando sus tasas de mortalidad $^{5-7}$. Hoy en día, numerosos países han seguido su ejemplo realizando reportes a nivel nacional, regional u hospitalario, destacando Suiza,
Nueva Zelanda, Japón, Canadá, Gales, Australia, Estados Unidos de Norteamérica, Arabia Saudita y Francia ${ }^{6,8-15}$. Los reportes de sobrevida más recientes corresponden a las estadísticas de mortalidad del Reino Unido y de Francia. Dodge ${ }^{5}$ reportó en 2006 la sobrevida de pacientes con FQ del Reino Unido hacia fines de 2003 según año de nacimiento y sexo, correspondiendo para los pacientes nacidos en 1978, $55 \%$ (varones) y $49 \%$ (mujeres); en 1988, $81 \%$ y $88 \%$, respectivamente y en 1992 , $97 \%$ y $96 \%$, respectivamente. La predicción de sobrevida para los pacientes nacidos en 2000 sobrepasa los 50 años. Por su parte, Bellis $^{16}$ reportó en 2006 la esperanza de vida de pacientes franceses con FQ entre 2001 y 2003 registrados en ONM (Observatoire National de la Mucoviscidose), los cuales alcanzan una sobrevida promedio de 39,1 años.

En Chile, un catastro realizado en 2002 demostró que existían sólo 9 equipos para realizar la prueba del sudor en el sistema público a nivel nacional, factor responsable en parte de un alto subdiagnóstico o bien de diagnóstico tardío. Ese mismo año se creó el Programa Nacional del Fibrosis Quística, cuyos principales objetivos fueron reducir el promedio de la edad del diagnóstico, incrementar el promedio de años de sobrevida y mejorar la calidad de vida de los pacientes. Hacia fines de 2003 el programa había identificado un total de 270 pacientes atendidos en el sistema público de salud, 55,7\% varones, de edades entre 3 meses y 34 años (media 10 años y 8 meses). La edad del diagnóstico osciló entre el primer mes de vida y los 12 años y 8 meses (promedio 6 años y 7 meses), bastante tardía en comparación a países desarrollados ${ }^{4}$. En 2003 se distribuyeron equipamiento e insumos para realizar el test de sudor en 28 hospitales del país, con lo cual se espera contribuir a reducir la tasa de subdiagnóstico y rebajar la edad promedio en que el diagnóstico se realiza.

Numerosos estudios han evaluado la influencia de diversos factores en la mortalidad de pacientes con FQ. En una cohorte de 21.047 
pacientes seguidos entre 1988 y 1992 en Estados Unidos de Norteamérica, se reportó un riesgo 1,6 veces mayor de fallecer en pacientes mujeres de edades entre 1 y 20 años. Más allá de dicho rango, la sobrevida no varió significativamente según sexo ${ }^{17}$. Asimismo, en una cohorte de 23.817 pacientes ajustada por diversas variables confundentes, se demostró que el grupo de menor ingreso económico familiar presentó un riesgo 1,4 veces mayor de fallecer por FQ, en comparación al grupo de mayor ingreso económico ${ }^{18}$. Por su parte, estudios de mortalidad según el tipo de mutación del gen que codifica para la proteína CFTR han demostrado que diferentes genotipos se asocian a fenotipos de mayor mortalidad, al igual que la condición de homocigoto versus heterocigoto para una misma mutación ${ }^{19,20}$. De igual forma, diversos grupos han evaluado el efecto de infecciones y de la respuesta inmune contra microorganismos como Burkholderia cepacia y Pseudomonas aeruginosa sobre la mortalidad de pacientes con $\mathrm{FQ}^{21-23}$. En una cohorte de 3.323 pacientes, se demostró un riesgo 2,6 veces mayor de fallecer en pacientes con cultivos positivos para Pseudomonas aeruginosa, en comparación a pacientes con cultivos negativos ${ }^{23}$. Otros factores condicionantes de mortalidad en pacientes con FQ tales como la función pulmonar ${ }^{24,25}$ y condición inmune ${ }^{26}$ también han sido estudiados, al igual que la mortalidad en diferentes escenarios tales como antes y después de un trasplante pulmonar ${ }^{27-31}$ y pacientes hospitalizados en unidades de cuidados intensivos ${ }^{32}$.

En Chile, la preocupación por describir aspectos epidemiológicos, clínicos y genéticos de esta enfermedad se inicia a fines de los años ochenta, destacando la descripción de Guiraldes ${ }^{33}$ y cols de la entonces considerada como enfermedad emergente en nuestro país y la comunicación de Lira ${ }^{34}$ sobre la Corporación para la Fibrosis Quística. Durante los años noventa se inicia la investigación epidemiológica, destacando dos estudios. El primero corresponde a la investigación liderada por Macri35, quien condujo en 1991 un estudio retrospectivo de 743 pacientes con FQ cubriendo el período 1979-89 en Argentina, Brasil, Chile y México. Considerando solamente datos chilenos, entre sus resultados destaca la edad promedio de defunción (3,1 años) y el tiempo de sobrevida de $50 \%$ de los pacientes al momento del diagnóstico
(4-5 años), vislumbrándose la necesidad de conducir futuras investigaciones epidemiológicas para dimensionar apropiadamente la magnitud de esta enfermedad. El segundo estudio corresponde a la experiencia de Soza ${ }^{36}$ y cols, quienes condujeron un estudio clínico, de confirmación diagnóstica, realizado íntegramente en Chile. Un total de 200 niños con sospecha clínica de FQ fueron sometidos a un test de sudor, confirmándose el diagnóstico de $\mathrm{FQ}$ en siete $(3,5 \%)$, cuya edad promedio era de 4,8 años.

Otras áreas relacionadas a esta patología también han sido estudiadas en Chile. Una importante línea de investigación se inició a mediados de los años noventa y corresponde a los estudios genéticos sobre FQ. Su principal orientación fue la identificación de los genotipos más frecuentes en nuestro país y la correlación genotipo-fenotipo ${ }^{37,38}$. A partir de 2000 se consolidan diversas líneas de investigación, que contribuyen al cuerpo de conocimientos sobre caracterización genotípica y expresión clínica de esta enfermedad ${ }^{39-43}$. El nuevo milenio se inició con un importante hito, cual fue la publicación del Consenso Nacional de Fibrosis Quística $^{3}$, trabajo multicéntrico conducido por las ramas de enfermedades respiratorias, genética, nutrición y gastroenterología de la Sociedad Chilena de Pediatría. El consenso resumió los principales aspectos epidemiológicos, diagnósticos y terapéuticos de esta patología, aportando lineamientos claros para la práctica médica. De igual forma, una nueva línea de investigación se iniciaba en Chile: el estudio de función pulmonar y ruidos respiratorios en pacientes con $\mathrm{FQ}^{44-45}$, permitiendo conocer con mayor profundidad los mecanismos fisiopatológicos subyacentes de esta enfermedad.

El futuro se proyecta con importantes desafíos. El proceso de transición epidemiológica avanzada que experimenta nuestro país, en el cual las enfermedades crónicas cobran una importancia relativa cada vez mayor, obliga a delinear nuevas estrategias de salud destinadas a garantizar el debido cuidado de pacientes portadores de patologías crónicas. Durante 2006 y 2007 numerosas entidades clínicas con Garantías Explícitas en Salud (GES) se incorporarán en forma progresiva al AUGE, entre ellas la FQ. De igual forma, a medida que las condiciones sanitarias del país experimentan una mejoría progresiva, habría de esperarse un aumento en la sobrevida de pacien- 
tes con FQ, con lo cual muchos de ellos alcanzarán la adultez. De hecho, se estima que $45 \%$ de los pacientes estadounidenses y europeos con FQ son mayores de 18 años $^{46}$. En consecuencia, surge la necesidad de contar con programas de transición debidamente diseñados, interdisciplinarios y que procuren no solamente aumentar la sobrevida de

\section{REFERENCIAS}

1. Vega-Briceño LE, Sánchez I. Fibrosis quística: Actualización en sus aspectos básicos. Rev Chil Pediatr 2005; 76: 464-70.

2. Ratjen F, Doring G. Cystic fibrosis. Lancet 2003; 361: 681-9.

3. SÁnchez I, Pérez Ma , Boza L, Lezana V, Vila Mạ Repetto G, et al. Consenso nacional de fibrosis quística. Rev Chil Pediatr 2001; 72: 356-80.

4. Astudilo P. Fibrosis quística y oxigenoterapia ambulatoria en Chile. Programas Nacionales. Boletín de Neumología Pediátrica 2004; 1: 11-6.

5. Dodge JA, Lewis PA, Stanton M, Wilsher J. Cystic Fibrosis mortality and survival in the United Kingdom, 1947 to 2003. Eur Respir J 2007; 29: 522-6.

6. Phelan P, Hey E. Cystic fibrosis mortality in England and Wales and in Victoria, Australia 1976-80. Arch Dis Child 1984; 59: 71-3.

7. Nielsen OH, Thomsen BL, Green A, Andersen PK, Hauge M, Schiotz PO. Cystic fibrosis in Denmark 1945 to 1985. An analysis of incidence, mortality and influence of centralized treatment on survival. Acta Paediatr Scand 1988; 77: 836-41.

8. Kraemer R, Stoll E, Moser H, Rossi E. Morbidity and mortality in cystic fibrosis. Results of 20 years of experience. Schweiz Med Wochenschr 1977; 107: 271-5.

9. Wesley AW, Stewart AW. Cystic fibrosis in New Zealand: incidence and mortality. NZ Med J 1985; 98: 321-3.

10. Robinson PG, EшоTt RB, Fraser J. Cystic fibrosis in New Zealand: incidence and mortality data. N Z Med J 1976; 83: 268-70.

11. Imaizumi $Y$. Incidence and mortality rates of cystic fibrosis in Japan, 1969-1992. Am J Med Genet 1995; 58: 161-8.

12. Corey M, Farewell V. Determinants of mortality from cystic fibrosis in Canada, 1970-1989. Am J Epidemiol 1996; 143: 1007-17. estos pacientes, sino también dotar de calidad estos años de vida. De igual forma, la implementación de estrategias de diagnóstico precoz, registros de casos nuevos y seguimiento de pacientes con FQ permitirá calcular cifras nacionales de incidencia y sobrevida, más confiables que las disponibles en la actualidad.

13. SiNGER RB. Cystic fibrosis mortality: registry data of cystic fibrosis. J Insur Med 1997; 29: 233-9.

14. Hawdburton CS, Mannino DM, Olney RS. Cystic fibrosis deaths in the United States from 1979 through 1991. An analysis using multiple-cause mortality data. Arch Pediatr Adolesc Med 1996; 150: 1181-5.

15. BANJAR H. Morbidity and mortality data of cystic fibrosis patients. Saudi Med J 2003; 24: 730-5.

16. Belus G, Cazes MH, Parant A, Gaimard M, Travers C, Le Roux E et AL. Cystic fibrosis mortality trends in France. J Cyst Fibros 2007; 6: 179-86.

17. Rosenfeid M, Davis R, Fitzsimmons S, Pepe M, Ramsey B. Gender gap in cystic fibrosis mortality. Am J Epidemiol 1997; 145: 794-803.

18. O'Connor GT, Quinton HB, Kneeland T, Kahn R, LEVER T, MADDock J ET AL. Median household income and mortality rate in cystic fibrosis. Pediatrics 2003; 111(4 Pt 1): e333-9.

19. Mckone EF, Emerson SS, Edwards KL, AitKen ML. Effect of genotype on phenotype and mortality in cystic fibrosis: a retrospective cohort study. Lancet 2003; 361: 1671-6.

20. Schibier A, Bolt I, Galiati S, Schoni MH, Kraemer R. High morbidity and mortality in cystic fibrosis patients compound heterozygous for 3905insT and deltaF508. Eur Respir J 2001; 17: 1181-6.

21. Fauroux B, Hart N, Belfar S, Boule M, TilousBorde I, Bonnet D ET AL. Burkholderia cepacia is associated with pulmonary hypertension and increased mortality among cystic fibrosis patients. J Clin Microbiol 2004; 42: 5537-41.

22. Moss RB, Hsu YP, Lewiston NJ, Curd JG, Milgrom $\mathrm{H}$, HART S ET AL. Association of systemic immune complexes, complement activation, and antibodies to Pseudomonas aeruginosa lipopolysaccharide and exotoxin A with mortality in cystic fibrosis. Am Rev Respir Dis 1986; 133: 648-52.

23. Emerson J, Rosenfeld M, Mcnamara S, Ramsey B, Gibson RL. Pseudomonas aeruginosa and other 
predictors of mortality and morbidity in young children with cystic fibrosis. Pediatr Pulmonol 2002; 34: 91-100.

24. Pianosi P, Leblanc J, Almudevar A. Peak oxygen uptake and mortality in children with cystic fibrosis. Thorax 2005; 60: 50-4.

25. Sharma R, Florea VG, Bolger AP, Doehner W, Florea ND, Coats AJ et al. Wasting as an independent predictor of mortality in patients with cystic fibrosis. Thorax 2001; 56: 746-50.

26. WisNiesKi JJ, TOdD EW, FuLER RK, Jones PK, DEARBORN DG, BoAT TF ET AL. Immune complexes and complement abnormalities in patients with cystic fibrosis. Increased mortality associated with circulating immune complexes and decreased function of the alternative complement pathway. Am Rev Respir Dis 1985; 132: 770-6.

27. Padila J, Calvo V, Jorda C, Escriva J, Cerón J, Penalver JC et al. Lung transplantation in cystic fibrosis: perioperative mortality. Arch Bronconeumol 2005; 41: 489-92.

28. Tantisira KG, Systrom DM, Ginns LC. An elevated breathing reserve index at the lactate threshold is a predictor of mortality in patients with cystic fibrosis awaiting lung transplantation. Am J Respir Crit Care Med 2002; 165: 1629-33.

29. Stanchina ML, Tantisira KG, Aquino SL, Wain JC, GinNs LC. Association of lung perfusion disparity and mortality in patients with cystic fibrosis awaiting lung transplantation. J Heart Lung Transplant 2002; 21: 217-25.

30. Augarten A, Akons H, Aviram M, Bentur L, Blau H, PICARD E ET AL. prediction of mortality and timing of referral for lung transplantation in cystic fibrosis patients. Pediatr Transplant 2001; 5: 33942.

31. Kerem E, Reisman J, Corey M, Canny GJ, Levison H. Prediction of mortality in patients with cystic fibrosis. N Engl J Med 1992; 326: 1187-91.

32. Texereau J, Jamal D, Choukroun G, Burgel PR, DieHL JL, RaBBat A ET AL. Determinants of mortality for adults with cystic fibrosis admitted in Intensive Care Unit: a multicenter study. Respir Res 2006; 7: 14 .

33. Guiraldes E, Valenzuela C, Tagle MS, Largo I. Fibrosis quística: una enfermedad emergente. Rev Méd Chile 1988; 116: 1336-8.

34. LiRA P, TAgle S. Corporación para la fibrosis quística. Rev Méd Chile 1989; 116: 587.
35. Macri C, De Gentile A, Manterola A, Tomezzol S, Reis F, Largo I et al. Epidemiology of cystic fibrosis in Latin America: preliminary communication. Pediatr Pulmonol 1991; 10: 249-53.

36. Soza G, Betancourt M, Hebel E, Álvarez M, Tapia N. Fibrosis quística del páncreas en la IX Región. Rev Chil Pediatr 1992; 63: 8-12.

37. Aspilaga M, Avendaño I, Largo I, Valenzuela C, Riveros N, Orellana O et al. Estudio genético molecular de la fibrosis quística en la población chilena. Relación con su expresión clínica. Rev Méd Chile 1993; 121: 1233-9.

38. Ríos J, Oreliana O, Aspilaga M, Avendaño I, Largo I, Rveros N. CFTR mutations in Chilean cystic fibrosis patients. Hum Genet 1994; 94: 291-4.

39. Molna G, González F, Cave R, Cornejo M, Navarro S, Deglin M et al. Estudio clínico-genético molecular de la fibrosis quística en la V Región, Chile. Rev Méd Chile 2002; 130: 850-8.

40. Vera A, Henríquez-Roldán C, González F, Molna G. Búsqueda de la mutación delta F508 y análisis de dos polimorfismos de nucleótido único en el gen CFTR, en una muestra de población general de Valparaíso, Chile. Rev Méd Chile 2005; 133: 767-75.

41. Riveros N, Rios J. Detección indirecta de portadores de fibrosis quística en dos familias chilenas mediante análisis de polimorfismos en el ADN asociados fuertemente al gen CFTR. Rev Méd Chile 2005; 133: 648-54.

42. Repetto G, Poggi H, Harris P, Navarro H, Sánchez I, GuiRALDES E ET AL. Identificación de mutaciones en el gen CFTR en pacientes chilenos con fibrosis quística. Rev Méd Chile 2001; 129: 841-7.

43. Navarro H, Kolbach R, Repetto G, Guiraldes E, HaRris P, Foradori A et al. Correlación genotipofenotipo de un grupo de pacientes con fibrosis quística. Rev Méd Chile 2002; 130: 475-81.

44. Sánchez I, Vizcaya C, García D. Análisis de los ruidos respiratorios y sibilancias en fibrosis quística. Rev Chil Pediatr 2003; 74: 590-4.

45. Sánchez I, Simonetti M, De Koster J, Chernick V. Efecto de la maniobra inspiratoria y de la hiperinsuflación pulmonar en los flujos espiratorios máximos en pacientes con fibrosis quística. Rev Chil Enf Respir 2003; 19: 15-20.

46. Vega-Briceño Le, Guiraldes E, Sánchez I. Fibrosis quística: enfrentando la transición desde el pediatra hacia el internista. Rev Méd Chile 2006; 134: 365-71. 